INTERVENTIONAL CARDIOLOGY AND SURGERY

\title{
Adverse events in coronary artery bypass graft (CABG) trials: a systematic review and analysis
}

\author{
L Nalysnyk, K Fahrbach, M W Reynolds, S Z Zhao, S Ross
}

Heart 2003;89:767-772

See end of article for authors' affiliations

Correspondence to: Dr L Nalysnyk, MetaWorks Inc, 10 President's Landing, Medford, Massachusetts 02155,

USA; LNalysnyk@ metawork.com

Accepted

19 February 2003

\begin{abstract}
Objectives: To quantify the incidence of major adverse events (AEs) occurring in hospital or within 30 days after surgery in patients undergoing coronary artery bypass graft (CABG) surgery and to identify risk factors for these AEs.

Methods: Systematic review and analysis of studies published in English since 1990. Studies of isolated standard CABG reporting postoperative incidence of myocardial infarction (MI), stroke, gastrointestinal bleeding, renal failure, or death in hospital or within 30 days were eligible for inclusion. Incidence of these events was calculated overall and for selected patient groups defined by all elective CABG versus mixed (some non-elective); mean ejection fraction $\leqslant 50 \%$ versus $>50 \%$; mean age $\leqslant 60$ versus > 60 years; primary CABG versus some reoperations; randomised controlled trials versus cohort studies; and single centre versus multicentre studies. Odds ratios of selected AEs were computed according to group risk factors.

Results: 176 studies (205 717 patients) met all inclusion criteria. The average incidence of major AEs occurring in-hospital was death (1.7\%); non-fatal MI (2.4\%); non-fatal stroke (1.3\%); gastrointestinal bleeding $(1.5 \%)$; and renal failure $(0.8 \%)$. Thirty day mortality was $2.1 \%$. Meta-analyses show that age $>70$, female sex, low ejection fraction, history of stroke, Ml, or heart surgery, and presence of diabetes or hypertension are all associated with increased 30 day mortality after CABG.

Conclusion: The incidence of major $A E s$ in patients after $C A B G$ varies widely across studies and patient populations, and this heterogeneity must be controlled when using the literature to benchmark safety.
\end{abstract}

M ore than 800000 patients undergo coronary artery bypass graft (CABG) surgery worldwide each year. CABG surgery continues to change, with a greater emphasis on arterial grafting, refinements in cardioplegia, and the introduction of minimally invasive surgery with or without the use of cardiopulmonary bypass. ${ }^{1}$ Although the overall results of CABG have improved in recent years, revascularisation of the heart is still associated with a risk of perioperative and postoperative death and morbidity. Patients undergoing $\mathrm{CABG}$ are now older and a larger number have had previous myocardial infarction (MI), stroke, or heart surgery. ${ }^{2}$ Consequently, morbidity and mortality after CABG surgery is expected to increase despite procedural advances.

The objective of this analysis was to quantify the incidence of early postoperative major adverse events (AEs) (MI, stroke, gastrointestinal bleeding, renal failure requiring dialysis, and death) in a standard CABG population. To achieve this objective, we systematically reviewed the recent published literature to build the evidence base and then performed incidence calculations for various groups of patients defined by both study and treatment group characteristics.

\section{METHODS}

In general, procedures for this review followed established best methods for the evolving science of systematic review research. ${ }^{34}$ A protocol was written prospectively, which stated the objectives, search criteria, study selection criteria, data elements of interest, and plans for analysis.

\section{Literature search}

We reviewed the English language literature from 1990 to May 2001. Our literature search consisted of computerised searches of Medline and Current Contents CD-ROMs using the combination of the following terms: coronary artery bypass or $C A B G$, clinical trials or cohort studies, human, and English. Bibliographies of all accepted studies and recent review papers were manually searched for additional potentially eligible citations.

We searched for studies of patients undergoing isolated standard CABG surgery in the past decade. To be included in the review, published studies had to report the incidence of postoperative MI, stroke, gastrointestinal bleeding, renal failure, or death occurring in hospital or within 30 days after surgery. Randomised controlled trials (RCTs) and non-RCTs, as well as cohort studies, were eligible. Since our objective was to quantify the incidence of these postoperative events in a standard CABG population, only "standard", "control" or "placebo" group outcomes were considered from RCTs or non-RCTs. To be eligible for inclusion in this review, RCTs or non-RCTs had to enrol at least 20 patients per group and observational cohort studies at least 50 patients.

We excluded studies that selected all patients with a particular history, risk factor, or comorbidities such as diabetes, previous MI, renal dysfunction, older age $(>70)$, poor ejection fraction $(<30 \%)$, unstable angina, reoperation, or emergency CABG. Such a study population was defined as having a high risk. However, studies that enrolled general populations containing some patients with these characteristics were included in this review. Studies comparing bypass surgical techniques or types of anaesthesia without a conventional CABG control group were excluded. Because our goal was to quantify only postoperative events, when a study

Abbreviations: $A E$, adverse event; $C A B G$, coronary artery bypass graft; $\mathrm{MI}$, myocardial infarction; n, number of patients; NYHA, New York Heart Association; RCT, randomised controlled trial; REM, random effects model 
reported perioperative outcomes and it was not clear that these occurred after surgery, the study was excluded from our review. Studies reporting only intraoperative events were also excluded.

\section{Database development}

Data from all accepted studies were extracted to a data form by one investigator, and all elements were reviewed and agreed upon by a second investigator before data entry. Data elements sought from each accepted study were protocol specified study, patient, and treatment characteristics, and the numbers of patients with AEs of interest. The timing of postoperative AEs reported was also sought as a categorical variable-that is, in hospital or within 30 days after surgery.

\section{Analysis}

The primary outcome of interest was the incidence of five specific AEs in CABG groups. For outcomes other than death, non-fatal events were sought, but if only total events (fatal and non-fatal) were reported, these were extracted and analysed separately. The average incidence of MI, stroke, gastrointestinal bleeding, renal failure, and death across studies was estimated by a random effects model (REM) meta-analytical technique ${ }^{5-7}$ using restricted maximum likelihood estimation. The REM assumes heterogeneity of the studies and weighs each study by both its within study sampling error and the estimated between study variation in $\mathrm{AE}$ incidence. When the between study variation is zero, the REM gives results identical to those of a fixed effects model. Only the REM results are reported, as it is generally a more conservative estimate.

The potential influence on incidence of AEs of study variables (location (North America, Europe, or other); number of study sites (single versus multicentre), study design (RCT versus cohort)) and group variables (timing of CABG (elective versus mixed); mean age ( $<60 v \geqslant 60$ years); ejection fraction $(\leqslant 50 \% v>50 \%$ ), and prior CABG (none $v$ some patients with reoperations)) was also studied. Bivariate metaregressions were conducted relating selected study characteristics to each of the individual AEs. The computed incidences are presented as mean (SE) and medians.

Many studies reported results stratified by sex, prior condition (for example, prior MI, prior stroke), or age. This allowed us to investigate more precisely the relations between patient level characteristics and the odds of a patient suffering a given AE. Where possible, odds ratios were calculated relating each possible patient characteristic to each reported AE; these odds ratios were meta-analysed using methods similar to those mentioned above.

All calculations were performed using SPSS software version 10.1 (SPSS Inc, Chicago, Illinois, USA) and SAS/IML software version 8.1 (SAS Institute, Cary, North Carolina, USA).

In the following results, " $\mathrm{k}$ " refers to the number of studies, " $t$ " to the number of treatment groups, and " $n$ " to the number of patients.

\section{RESULTS \\ Studies}

The literature search including manual bibliography checks yielded 4885 citations. The vast majority were rejected immediately for reasons such as ineligible language or patient population. Full papers were retrieved for 596 abstracts that had no apparent reason for exclusion. Of these, 388 were subsequently rejected for reasons of absence of reporting of AEs of interest, unclear timing of AEs, or mixing of data of patients undergoing $\mathrm{CABG}$ and data of patients having valve replacement or other concomitant procedures, or because only late (after 30 days) postoperative AEs were reported. In addition, several publications reported on the same patient population (kin studies), and these were extracted as one study to avoid double counting of results. Therefore, 176 primary and 32 kin studies were found to satisfy all inclusion criteria and were included in this review. A citation list of 176 primary studies is provided in the appendix on the Heart website.

The accepted studies were conducted in Europe $(\mathrm{k}=54$, $\mathrm{n}=34437)$ and North America $(\mathrm{k}=91, \mathrm{n}=154524)$, and $31(n=16706)$ were multinational trials or studies conducted in other geographic locations. There were 69 RCTs $(\mathrm{n}=9598), 13$ non-RCTs $(\mathrm{n}=2019)$, and 94 cohort studies $(\mathrm{n}=194050)$. The majority of studies were conducted in a single centre $(\mathrm{k}=146, \mathrm{n}=105104)$ and 30 studies $(\mathrm{n}=100563)$ were multicentre trials evaluating in total approximately the same number of patients as all the single centre studies. Most of the studies were published very recently, after $1995(\mathrm{k}=135)$.

\section{Patient and treatment characteristics}

Table 1 summarises baseline and operative patient characteristics. There were 176 treatment groups overall, comprising 205667 patients. The majority of patients in all studies were men $(81.6 \%)$. The average age of all patients ranged from $35-71.3$ years with the overall mean being 62.8 years. In the 31 studies reporting New York Heart Association (NYHA) classification, more than half of all patients $(67.6 \%)$ were in NYHA class III or IV. Approximately $10 \%$ of patients in this set of studies had low left ventricular ejection fraction $(<35-40 \%)$.

Table 1 also shows the prevalence of comorbid conditions and risk factors among patients in these studies. More than $50 \%$ of patients in groups reporting this information had hypertension or high cholesterol concentration $(52.1 \%$ and $54.2 \%$, respectively). Previous MI was reported by $46.7 \%$ and unstable angina by $32.1 \%$ of patients in groups reporting these histories. There was also a significant proportion of patients who had previous CABG or revascularisation $(5.8 \%$ and $6.1 \%$, respectively).

CABG surgery was urgent or an emergency in $28 \%$ of patients in 115 studies. During CABG, patients received an average of 3.2 grafts (range of means 1-4.5). Only two studies were single graft studies.

\section{In-hospital adverse events}

Table 2 displays the incidences of in-hospital AEs. MI, both all MIs and non-fatal MIs, was the most prevalent AE in the overall CABG population and across all stratified categories. Because of the various diagnostic criteria used to define MI, the incidence of $M I$ differs widely across the studies, from 0-29.2\% with the average of $3.9 \%$ (median $2.9 \%$ ). In five studies (all RCTs) it was greater than 10\% (Carrier 1998, Menashe 1995, Multicenter Study of Perioperative Ischemia 1995, MullisJansson 1999, and Searle 1996). The incidence of MI differed significantly between RCTs and cohort studies (6.3\% v 2.7\%, $\mathrm{p}<0.05)$ and between single centre and multicentre studies $(2.8 \% \vee 7.9 \%, \mathrm{p}<0.01)$. A greater incidence of MI was found in studies enrolling patients with prior CABG than in those without prior CABG $(6.5 \% \vee 2.7 \%)$, but this did not reach significance. Non-fatal MI occurred on average in $2.4 \%$ (median $2.4 \%$, range $0-13.9 \%$ ) of the overall CABG patients and was slightly lower in elective CABG than in mixed $(2.3 \% v 2.6 \%)$. Interestingly, older age ( $>60$ years) and lower mean ejection fraction $(\leqslant 50 \%)$ were not associated with a higher incidence of MI.

Non-fatal strokes occurred in $1.3 \%$ (median 1.3\%, range $0-3.2 \%)$ of patients and the incidence was lower in groups with elective surgery $(1.0 \% v 1.5 \%)$ and no prior CABG $(1.0 \%$ $v 1.8 \%)$, and it was significantly lower in RCTs than in cohort studies $(1.0 \% v 1.5 \%, \mathrm{p}<0.01)$. Surprisingly, in studies that did not distinguish between fatal and non-fatal strokes (strokes), the rate appeared to be higher in younger groups (mean age $\leqslant 60$ years, $2.8 \% \vee 1.9 \%$ ), but it was not significant. 
Table 1 Patient characteristics

\begin{tabular}{|c|c|c|c|}
\hline & k & $n$ & $\%$ \\
\hline Total & 176 & 205667 & 100 \\
\hline Mean age & 176 & 165578 & Mean 62.8 years \\
\hline Age $>70$ & 25 & 48839 & $30.1 \%$ \\
\hline $\operatorname{Sex}(M / F)$ & 168 & $161076 / 36441$ & $81.6 \% / 18.4 \%$ \\
\hline NYHA class III-IV & 31 & 74674 & $67.6 \%$ \\
\hline Mean LVEF & 83 & 52563 & $55.1 \%$ \\
\hline Low ejection fraction (<35-40\%) & 53 & 108954 & $9.9 \%$ \\
\hline \multicolumn{4}{|l|}{ Comorbidities } \\
\hline Diabetes & 118 & 174049 & $24.6 \%$ \\
\hline Renal dysfunction & 49 & 75488 & $5.0 \%$ \\
\hline Hypertension & 95 & 78783 & $52.1 \%$ \\
\hline History of stroke or CVD & 54 & 92662 & $11.5 \%$ \\
\hline Previous Ml & 91 & 118897 & $46.7 \%$ \\
\hline Previous $C A B G /$ heart surgery & 101 & 158638 & $5.8 \%$ \\
\hline Previous revascularisation (PTCA) & 21 & 33499 & $6.1 \%$ \\
\hline Unstable angina & 40 & 45896 & $32.1 \%$ \\
\hline Hypercholesterolaemia & 39 & 39372 & $54.2 \%$ \\
\hline History of smoking & 57 & 92937 & $36.6 \%$ \\
\hline \multicolumn{4}{|l|}{ Operative data } \\
\hline Urgent/emergency CABG & 115 & 158719 & $28.1 \%$ \\
\hline Warm blood cardioplegia & 65 & 29293 & $19.8 \%$ \\
\hline Mean number of grafts & 111 & 69128 & 3.2 (range 1-4.5) \\
\hline
\end{tabular}

Gastrointestinal bleeding was reported by only eight studies. Overall, the incidence of gastrointestinal bleeding after CABG among 12897 patients was $1.5 \%$ (median $1.2 \%$, range $0.7-2.7 \%)$. Patients without prior CABG appeared to have a higher incidence of gastrointestinal bleeding than the groups where some patients had prior bypass surgery $(2.6 \% v 1.5 \%)$. But, with so few studies, no differences were significant in the incidence of gastrointestinal bleeding with respect to any of the variables assessed.

The incidence of renal failure requiring dialysis was low. In 23 studies $(\mathrm{n}=22798)$ on average $0.8 \%$ (median $0.7 \%$, range $0-6.2 \%$ ) of patients needed dialysis after CABG. The frequency was higher in studies enrolling patients with urgent or emergency CABG than in elective CABG only $(0.9 \% v 0.5 \%)$ and it was significantly higher in cohort studies than in RCTs $(1.0 \%$ $v 0.4 \%, \mathrm{p}<0.05)$.

Postoperative mortality rate during hospital stay was 1.7\% (median $1.5 \%$ ) in the overall CABG population and ranged from $0-6.6 \%$. When studies were stratified as elective versus mixed (including patients with urgent or emergency CABG), the mortality rate was significantly lower in the elective CABG $(1.5 \% v 1.8 \%, p<0.05)$. In-hospital mortality rate was higher in studies enrolling older patients (mean age $>60$ years, $1.8 \%$ $v 1.2 \%)$ and in patients with no history of prior CABG $(1.2 \% v$ $1.9 \%)$. A significantly lower incidence of death was also noted in RCTs than in cohort studies $(1.5 \% v 1.8 \%, \mathrm{p}<0.05)$ and in single centre studies than in multicentre studies ( $1.5 \% \vee 2.5 \%$, $\mathrm{p}<0.05)$.

\section{Day adverse events}

Except for deaths, AEs at 30 days after surgery were not commonly reported. There were 70 studies that reported a mortality rate for death in hospital or within 30 days after surgery. An average $2.1 \%$ (median $2.0 \%$, range $0-7.7 \%$ ) of all CABG patients died within 30 days (table 2). In the overall CABG category the 30 day mortality rate was higher than the overall in-hospital death rate $(2.1 \% \vee 1.7 \%)$. Among stratified categories 30 day mortality incidence differed significantly with respect to number of study sites (single versus multicentre), elective versus mixed CABG groups, and mean age ( $\leqslant 60$ or $>60$ years), with the greater incidence in the latter category in each instance.
The 30 day outcomes for MI, stroke, gastrointestinal bleeding, and renal failure were reported too infrequently; therefore, a meta-analysis of the incidence of these events was not performed.

\section{Sensitivity analysis}

Since the incidence of major AEs after CABG surgery appears to differ in RCTs and cohort studies, we performed similar stratified analyses for all outcomes of interest separately for RCTs and cohort studies. These findings were consistent with the overall analysis of the incidence of major AEs after CABG and further confirmed the potential influence of some study (geographic location, number of study centres) and group variables (elective CABG only versus some patients with urgent of emergency $C A B G$, some patients with a history of prior $\mathrm{CABG}$ versus primary $\mathrm{CABG}$ ).

The potential impact of cardiopulmonary bypass duration on patient outcomes was tested in the meta-regression analysis. A weak but significant positive relation $(p=0.02)$ was noted between cardiopulmonary bypass duration and incidence of non-fatal MI. There was no significant relation between cardiopulmonary bypass duration and any of the other outcomes. It should be noted that the lack of significant findings does not imply that there is no relation between cardiopulmonary bypass duration and increased risk of AEs at the patient level. The metaregression can only investigate the study level relation between mean cardiopulmonary bypass duration and incidence of AEs, and thus this investigation has much lower power than an individual patient analysis would have.

\section{Analysis of risk factors}

In the set of 176 studies accepted in this review, there were 22 studies in which either risk factor odds ratios were reported for the AEs of interest or the information on these was available and it was possible to calculate the odds ratio of interest. Table 3 shows the individual study odds ratios and metaanalytical results for 30 day mortality by risk factors. These results clearly suggest that old age, female sex, presence of diabetes and hypertension, and history of prior heart surgery and MI are associated with increased risk of death after CABG. Except for diabetes (too few studies), the same is true for 


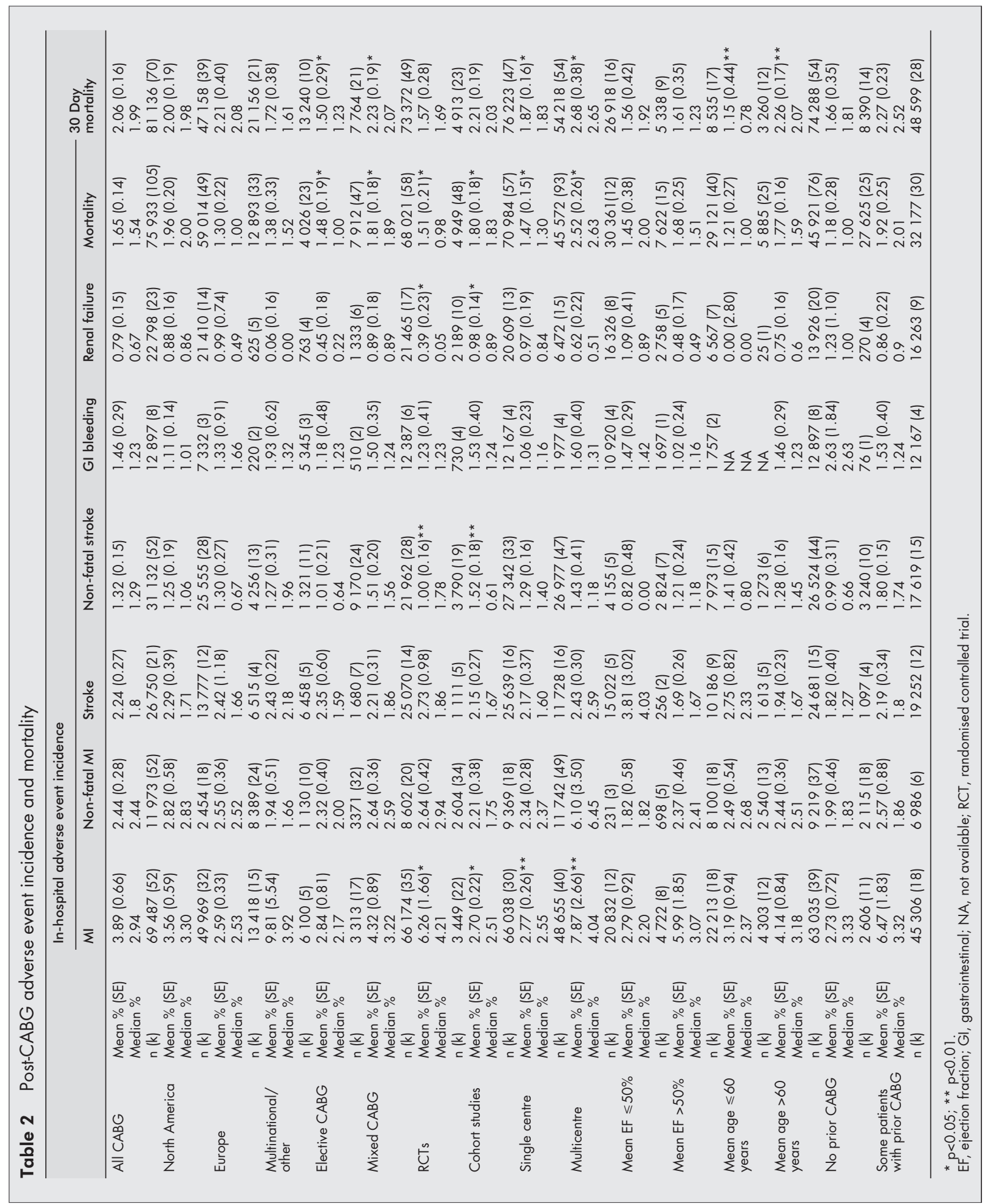

stroke (data not shown). The data were too sparse to perform similar meta-analyses for MI and renal failure; however, evidence suggests that female sex, age, and diabetes are positively related to incidence of renal failure after CABG.

\section{DISCUSSION}

This systematic review and exploration of early postoperative AEs in 176 conventional CABG surgery studies of more than 200000 patients shows that the incidence of in-hospital death and major AEs varies highly depending on study features such as year, number of centres involved, and study design, as well as patient group characteristics such as age, prior CABG, and timing of operation (elective only or mixed). These differences have now been quantified and must be recognised when interpreting AEs in individual CABG trials and when planning such studies.

The impact of study design was of particular interest in view of the recent debate on the relative merits of RCTs and observational studies. ${ }^{8-10}$ The much higher incidence of MI in RCTs than in cohort studies may be explained by closer monitoring of patients and more sensitive and aggressive detection methods in RCTs. The lower incidence of non-fatal stroke, 
Table 3 Odds ratios for 30 day mortality after CABG

\begin{tabular}{|c|c|c|c|}
\hline Risk factor & Study (year) & Odds ratio & $95 \% \mathrm{Cl}$ \\
\hline Female versus male & $\begin{array}{l}\text { Barbir (1994) } \\
\text { Brandup (1995) } \\
\text { Capdeville (2001) } \\
\text { Corbineau (1999) } \\
\text { Elisheva (2000) } \\
\text { Mesh (1997) } \\
\text { Rao (1996) } \\
\text { Wong (1999) } \\
\text { Meta-analysis result (REM, k=8) }\end{array}$ & $\begin{array}{l}3.10 \\
2.75 \\
2.07 \\
1.38 \\
2.01 \\
1.67 \\
1.37 \\
3.91 \\
1.92\end{array}$ & $\begin{array}{l}(0.88 \text { to } 10.89) \\
(1.62 \text { to } 4.68) \\
(1.59 \text { to } 10.33) \\
(0.86 \text { to } 2.21) \\
(1.43 \text { to } 2.83) \\
(0.61 \text { to } 4.58) \\
(0.91 \text { to } 2.07) \\
(1.68 \text { to } 9.08) \\
(1.49 \text { to } 2.48)\end{array}$ \\
\hline Diabetes versus no diabetes & $\begin{array}{l}\text { Brandup (1995) } \\
\text { Corbineau (1999) } \\
\text { Del Rizzo (1998) } \\
\text { Mesh (1997) } \\
\text { Rao (1996) } \\
\text { Meta-analysis result (REM, k=5) }\end{array}$ & $\begin{array}{l}2.11 \\
1.76 \\
1.76 \\
0.40 \\
1.93 \\
1.57\end{array}$ & $\begin{array}{l}(1.12 \text { to } 3.96) \\
(0.96 \text { to } 3.23) \\
(0.98 \text { to } 3.15) \\
(0.15 \text { to } 1.06) \\
(1.30 \text { to } 2.86) \\
(0.88 \text { to } 2.80)\end{array}$ \\
\hline Age $>70$ versus $\leqslant 70$ & $\begin{array}{l}\text { Brandup (1995) } \\
\text { Corbineau (1999) } \\
\text { Del Rizzo (1998) } \\
\text { Elisheva (2000) } \\
\text { Rao (1996) } \\
\text { Meta-analysis result (FEM, k=5) }\end{array}$ & $\begin{array}{l}1.84 \\
2.13 \\
2.33 \\
2.48 \\
3.07 \\
2.42\end{array}$ & $\begin{array}{l}(1.05 \text { to } 3.22) \\
(1.43 \text { to } 3.16) \\
(1.32 \text { to } 4.11) \\
(1.79 \text { to } 3.43) \\
(2.09 \text { to } 4.50) \\
(2.00 \text { to } 2.80)\end{array}$ \\
\hline $\begin{array}{l}\text { Prior heart surgery versus no } \\
\text { prior heart surgery }\end{array}$ & $\begin{array}{l}\text { Corbineau (1999) } \\
\text { Del Rizzo (1998) } \\
\text { Elisheva (2000) }\end{array}$ & $\begin{array}{l}3.51 \\
7.05 \\
1.32\end{array}$ & $\begin{array}{l}(1.60 \text { to } 7.69) \\
(3.21 \text { to } 15.47) \\
(0.61 \text { to } 2.86)\end{array}$ \\
\hline Prior $\mathrm{Ml}$ versus no prior $\mathrm{Ml}$ & $\begin{array}{l}\text { Brandup (1995) } \\
\text { Corbineau (1999) } \\
\text { Rao (1996) }\end{array}$ & $\begin{array}{l}1.69 \\
1.56 \\
1.93\end{array}$ & $\begin{array}{l}(0.96 \text { to } 2.98) \\
(1.05 \text { to } 2.32) \\
(1.25 \text { to } 2.99)\end{array}$ \\
\hline $\begin{array}{l}\text { Hypertension versus no } \\
\text { hypertension }\end{array}$ & $\begin{array}{l}\text { Brandup (1995) } \\
\text { Elisheva }(2000) \\
\text { Rao (1996) }\end{array}$ & $\begin{array}{l}1.73 \\
1.70 \\
1.71\end{array}$ & $\begin{array}{l}(1.03 \text { to } 2.89) \\
(1.20 \text { to } 2.40) \\
(1.15 \text { to } 2.54)\end{array}$ \\
\hline
\end{tabular}

gastrointestinal bleeding, and renal failure in the RCTs may be explained by the exclusion of high risk patients. In this review the majority of RCTs excluded patients with prior CABG, low ejection fraction, history of stroke or cerebrovascular disease, or age over 75 years. On the other hand, the large observational studies were usually performed on consecutive patients undergoing CABG in a single institution over a defined period of time. These cohort studies were expected to include a higher proportion of high risk patients, perhaps providing more "real world" results.

Furthermore, differences in AEs associated with differences in operative, anaesthetic, and postoperative techniques were not quantified in this review but should also be considered. What is "standard" or "conventional" CABG varies not just by year but by each centre and surgeon. We observed differences in the type of cardioplegia (warm or cold), bypass time, number and types of conduits used, time in the intensive care unit, extubation practices (early or late), and pain management, all of which may affect AE rates in the postoperative period. Ancillary medications surely differ also, especially with respect to use of antiplatelet agents, heparin, and anticoagulants, but these details were not typically reported. These factors may well contribute to the considerable variation in the reported incidence of major AEs, even when controlling for study and patient characteristic that are reported. Furthermore, improved outcomes after cardiac surgery as a result of better anaesthesiological and perfusion management may be offset by changing patient demographics-namely, the increasing age of the patients and higher prevalence of severe comorbidities, which are associated with an increased risk for early postoperative mortality and morbidity. ${ }^{11}$

Additional caveats arise from problems we encountered in many studies with definitional variability and uncertainty. For example, when MI was defined on the basis of ECG or enzyme criteria the incidences were comparable across studies. However, studies using stricter criteria for MI definition requiring both ECG and enzyme criteria reported a lower incidence of MI. In addition to different definitions of events across studies, we encountered many studies that had to be excluded from the review with unclear definitions. Important baseline patient characteristics were occasionally missing and these studies could not be used in the stratified and regression analyses. Terms that were frequently used and rarely defined were "perioperative", "urgent" and "emergent", "stroke", and "renal failure", to name a few.

Lastly, aside from providing a benchmark for AE incidences, this systematic review reinforces the need for future trialists to adopt uniform definitions and terminology, and always to provide a minimum base set of risk factors, perhaps those identified by the Society of Thoracic Surgeons. ${ }^{11}$

\section{ACKNOWLEDGEMENTS}

The authors gratefully acknowledge the expert assistance provided by the members of the MetaWorks CABG Review Group: Janet Connelly BS, Rhonda Estok RN BSN, Diana Frame MEM, Sidney Klawansky MD PhD, Cindy Levine MD, Veronica Ludensky BA, Tara McHugh MA, Melissa Privetera BS, Michael Rozinsky BA, Rachel Scheye BA, Jay Sethi, and Linna Slobodskaya BA.

Funded by Pharmacia Corporation, Peapack, New Jersey, USA.

\section{Authors' affiliations}

L Nalysnyk, K Fahrbach, S Ross, MetaWorks CABG Review Group, MetaWorks Inc, Medford, Massachusetts, USA

M W Reynolds, S Z Zhao, Pharmacia Corporation, Peapack, New Jersey, USA 


\section{REFERENCES}

1 Pepper J. Severe morbidity after coronary artery surgery. Curr Opin Cardiol 2000; 15:400-5.

2 Mangano DT, Siciliano D, Hollenberg $M$, et al. Postoperative myocardial ischemia: therapeutic trials using intensive analgesia following surgery. The study of perioperative ischemia (SPI) research group. Anesthesiology 1992;76:342-53.

3 Cook DJ, Mulrow CD, Haynes RB. Systematic reviews: synthesis of best evidence for clinical decisions. Ann Intern Med 1997;126:376-80.

4 Mulrow CD, Oxman AD, eds. Cochrane collaboration handbook. The Cochrane Library. The Cochrane Collaboration, Issue 1. Oxford: Update Software, 1997.

5 DerSimonian R, Laird N. Meta-analysis in clinical trials. Control Clin Trials 1986;7:177-88.

6 Hedges L, Olkin I. Statistical methods for meta-analysis. Orlando: Academic Press, 1985:230-57.

7 Bryk A, Raudenbush S. Hierarchical linear models. Newebury Park: Sage Publications, 1994.
8 Benson $\mathrm{K}$, Hurtz AJ. A comparison of observational studies and randomized controlled trials. N Engl J Med 2000;342: 1878-86.

9 Concato J, Shah N, Horwitz RI. Randomized controlled trials, observational studies, and the hierarchy of research designs. N Engl J Med 2000:342:1887-92.

10 Ernst E, Pittler MH. Assessment of therapeutic safety in systematic reviews: literature review. BMV 2001;323:546.

11 Society of Thoracic Surgeons. List of variables for prediction of operative deaths for 1996 coronary artery bypass-only procedures [online]. 7 April 2000. ttp://www.ctsnet.org/doc/4314.

To see the appendix comprising the 176 primary studies go to the Heart websitewww.heartinl.com/supplemental

\section{IMAGES IN CARDIOLOGY}

\section{Transcatheter closure of large fistula between left main coronary artery and right atrium using Amplatzer duct occluder}

A 4 year old girl was admitted with cyanosis after exercise. Physical examination found a continuous murmur which was loudest in the upper right parasternal region. The ECG was normal. Transthoracic echocardiography revealed mild right ventricular and atrial dilatation; it also revealed a fistula originating from the left main coronary artery (LMCA), rounding the ascending aorta and left atrium, and emptying into the right atrium. Ascending aorta angiography showed the presence of an aneurysmal fistula originating from the left main coronary artery and draining into the right atrium. The diameter of the fistulous orifice was $3.0 \mathrm{~mm}$ (upper panel, middle column).

A 5 French JL3.0 angiography catheter was introduced into LMCA. Within the catheter a 0.014 inch guiding wire was inserted into left main coronary artery and crossed the fistula into the right atrium. The guiding wire was caught in the right atrium by the Amplatz "goose neck" snare, and passed through a femoral vein. Over the wire a Mullins sheath was transvenously advanced across the fistula (lower panel, middle column). Then the guiding wire was removed. An 8/6 mm Amplatzer duct occluder (ADO) was advanced within the sheath until it reached the tip. The delivery system was carefully withdrawn until the ADO was opened completely at the atrial end of the fistula (upper panel, right column). Postdeployment angiography showed there was no residual shunting, and the coronary blood flow improved (lower panel, right). The murmur disappeared.
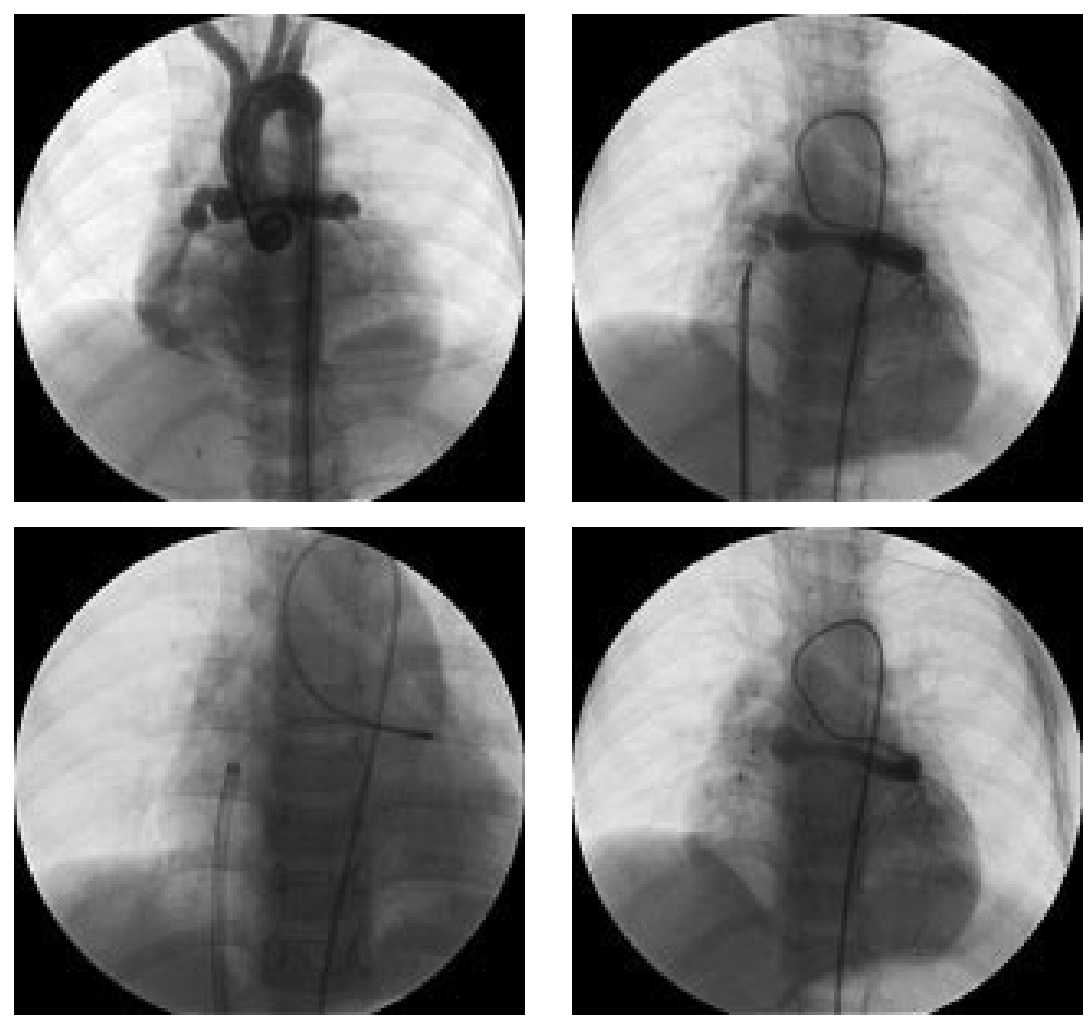

A coronary artery fistula most commonly originates from the right coronary artery, but a fistula originating from the left main coronary artery is rare. Coronary artery fistulae can cause myocardial ischaemia, congestive heart failure, bacterial endocarditis, cardiac arrhythmia, and rupture of aneurysmal fistulae. Treatment options include surgical ligation and coil embolisation. An Amplatzer duct occluder provides another means of treatment.

\section{J J Tang}

S H Zhou

$X$ Q Shen tangjianjun2002@hotmail.com 